\title{
HILBERT'S TENTH PROBLEM FOR A CLASS OF RINGS OF ALGEBRAIC INTEGERS
}

\author{
THANASES PHEIDAS
}

(Communicated by Thomas J. Jech)

\begin{abstract}
We show that $\mathbf{Z}$ is diophantine over the ring of algebraic integers in any number field with exactly two nonreal embeddings into $\mathbf{C}$ of degree $\geq 3$ over $\mathbf{Q}$.
\end{abstract}

Introduction. Let $R$ be a ring. A set $S \subset R^{m}$ is called diophantine over $R$ if it is of the form $S=\left\{x \in R^{m}: \exists y \in R^{n} p(x, y)=0\right\}$, where $p$ is a polynomial in $R[x, y]$. A number field is a finite extension of the field $\mathbf{Q}$ of rational numbers. If $K$ is a number field, we denote by $O_{K}$ the ring of elements of $K$ which are integral over the ring $\mathbf{Z}$ of rational integers.

$\mathbf{N}$ is the set $\{0,1,2, \ldots\}$ and $\mathbf{N}_{0}$ is the set $\{1,2,3, \ldots\}$.

In this paper we prove

THEOREM. Let $K$ be a number field of degree $n \geq 3$ over $\mathbf{Q}$ with exactly two nonreal embeddings into the field $\mathbf{C}$ of complex numbers. Then $\mathbf{Z}$ is diophantine over $O_{K}$.

An example of such a number field is $\mathbf{Q}(d)$ where $d^{3}$ is a rational number which does not have a rational cube root.

In order to prove the theorem, we use the methods of J. Denef in [3]. The terminology and enumeration of the lemmas is kept the same as in [3] so that the similarities and differences of the proofs are clear. The theorem implies

COROLlary. Let $K$ be as in the theorem. Then Hilbert's Tenth Problem in $O_{K}$ is undecidable.

The results of $[\mathbf{3}]$ and the present paper are the maximum that can be achieved using the present methods. Hence the general conjecture made in [4], namely that Hilbert's Tenth Problem for the integers of any number field is undecidable, remains open.

Let $K$ be a number field of degree $n \geq 3$ over $\mathbf{Q}$ with exactly two nonreal embeddings into $\mathbf{C}$. Let $\sigma_{i}, i=1,2, \ldots, n$, be all the embeddings of $K$ into $\mathbf{C}$, enumerated in such a way that $\sigma_{n-1}$ and $\sigma_{n}$ are nonreal. Then the embedding $\sigma: K \rightarrow \mathrm{C}$ such that $\sigma(x)=\overline{\sigma_{n}(x)}$ is distinct from $\sigma_{n}$ and from all $\sigma_{i}, i \leq n-2$,

Received by the editors July $16,1987$.

1980 Mathematics Subject Classification (1985 Revision). Primary 03B25; Secondary 12B99.

I would like to thank Professor Leonard Lipschitz for his encouragement and help during the preparation of this work.

In the process of publication of this paper I was informed that Alexandra Shlapentokh obtained the same results as part of her thesis at Courant Institute of Mathematical Sciences.

This paper has been supported in part by NSF Grant \#DMS 8605-198. 
since $\sigma_{n}$ is nonreal (i.e. for at least an $x \in K, \sigma_{n}(x) \notin \mathbf{R}$, hence $\sigma(x) \neq \sigma_{n}(x)$ and $\sigma(x) \notin \mathbf{R})$. Hence $\sigma=\sigma_{n-1}$ and therefore, for every $x \in K, \sigma_{n-1}(x)=\overline{\sigma_{n}(x)}$. In the rest of the paper we identify $K$ with $\sigma_{1}(K)$.

There are two cases: $\sigma_{n-1}(K)=\sigma_{n}(K)$ or $\sigma_{n-1}(K) \neq \sigma_{n}(K)$. In the first case, let $b$ be an element of $K$ such that $K=\mathbf{Q}(b)$. We have that $\operatorname{Re} \sigma_{n}(b) \in \sigma_{n}(K)$ and $\left(\operatorname{Im} \sigma_{n}(b)\right)^{2} \in \sigma_{n}(K)$ where $\operatorname{Re} x$ and $\operatorname{Im} x$ are the real and imaginary parts of $x$, respectively. So, since $\sigma_{n}(K)=\mathbf{Q}\left(\sigma_{n}(b)\right),\left[\sigma_{n}(K): \sigma_{n}(K) \cap \mathbf{R}\right]=2$ and so $\sigma_{n}(K)$ is nontotally real of degree 2 over $\sigma_{n}(K) \cap \mathbf{R}$ which is totally real. By [3] $\mathbf{Z}$ is diophantine over $\sigma_{n}\left(O_{K}\right) \cap \mathbf{R}$ and by the results of [4] this implies that $\mathbf{Z}$ is diophantine over $\sigma_{n}\left(O_{K}\right)$. Hence $\mathbf{Z}$ is diophantine over $O_{K}$. Therefore, we will consider only the case where $\sigma_{n-1}(K) \neq \sigma_{n}(K)$.

Let $a \in O_{K}$ be such that

$$
\left|\sigma_{i}(a)\right|<1 / 2^{4 n} \quad \text { for } i=1,2, \ldots, n-2 \text { and } a \neq 0 .
$$

For each $x \in O_{K}$, let $\delta(x) \in C$ be a number so that $\delta^{2}(x)=x^{2}-1$. Let $\delta=\delta(a)$ and call $L=K(\delta)$. By $(*) a$ may not be a rational integer and therefore $\delta \notin K$. So $[L: K]=2$ and each embedding $\sigma_{i}$ of $K$ into $\mathbf{C}$ extends to two embeddings $\sigma_{i, 1}$ and $\sigma_{i, 2}$ of $L$ into $\mathbf{C}$. The relations $\sigma_{i, 2}(\delta)=-\sigma_{i, 1}(\delta)$ are obvious. Call $\varepsilon=\delta+a$ and $x_{m}$ and $y_{m}$ the solutions in $O_{K}$ of the equation $x_{m}+\delta y_{m}=(a+\delta)^{m}$ for $m \in \mathbf{Z}$. Clearly $\varepsilon^{m}=x_{m}+\delta y_{m}, \varepsilon^{-m}=x_{m}-\delta y_{m}$, and $\varepsilon$ is a unit in $O_{L}$.

LEMMA 1. Let $K$ be any number field, and $a, b, c \in O_{K}$. Suppose $\delta(a), \delta(b) \notin$ $K$. Let $m, h, k, j \in N$. We have:

(1) $\varepsilon$ is a unit in $O_{K(\delta)}, \varepsilon^{-1}=a-\delta$, and $x_{m}, y_{m}$ satisfy the Pell equation $x^{2}-\left(a^{2}-1\right) y^{2}=1$;

(2) $x_{m}=\left(\varepsilon^{m}+\varepsilon^{-m}\right) / 2, y_{m}=\left(\varepsilon^{m}-\varepsilon^{-m}\right) / 2 \delta$;

(3) $x_{m \pm k}=x_{m} x_{k} \pm\left(a^{2}-1\right) y_{m} y_{k}, y_{m \pm k}=x_{k} y_{m} \pm x_{m} y_{k}$;

(4) $h\left|m \Rightarrow y_{m}\right| y_{h}$;

(5) $y_{h k} \equiv k x_{h}^{k-1} y_{h} \bmod y_{h}^{3}$;

(6) $x_{m+1}=2 a x_{m}-x_{m-1}, y_{m+1}=2 a y_{m}-y_{m-1}$;

(7) $y_{m}(a) \equiv m \bmod (a-1)$;

(8) if $a \equiv b \bmod c$, then $x_{m}(a) \equiv x_{m}(b) \bmod c$ and $y_{m}(a) \equiv y_{m}(b) \bmod c$;

(9) $x_{2 m \pm j} \equiv-x_{j} \bmod x_{m}$;

(10) if $n \in O_{K}$ and $n \neq 0$, then there exists an $m \in N_{0}$ such that $n \mid y_{m}(a)$.

PROOF. See [3].

LEMMA 2. Let a be as above. Then:

(1) for $i \leq n-2,0<\left|\sigma_{i}(a)\right|<1 / 2^{4 n}$ and $\left|\sigma_{n}(a)\right|=\left|\sigma_{n-1}(a)\right|>2^{2 n}$;

(2) for $i \leq n-2, j=1,2,\left|\sigma_{i, j}(\varepsilon)\right|=1$;

(3) $\left|\sigma_{n-1, j}(\varepsilon)\right| \neq 1$ and $\left|\sigma_{n, j}(\varepsilon)\right| \neq 1$ and

$$
\max \left\{\left|\sigma_{n, 1}(\varepsilon)\right|,\left|\sigma_{n, 2}(\varepsilon)\right|\right\}=\max \left\{\left|\sigma_{n-1,1}(\varepsilon)\right|,\left|\sigma_{n-1,2}(\varepsilon)\right|\right\}>2^{2 n} .
$$

PROOF. (1) Since $\sigma_{n-1}(a)=\overline{\sigma_{n}(a)},\left|\sigma_{n-1}(a)\right|=\left|\sigma_{n}(a)\right|$. Moreover $N_{K / \mathbf{Q}}(a)$ is a rational integer different from zero and hence $\prod_{i=1}^{n}\left|\sigma_{i}(a)\right|=\left|N_{K / \mathbf{Q}}(a)\right| \geq 1$. Since for $i \leq n-2,\left|\sigma_{i}(a)\right|<1 / 2^{4 n}$ we get $\left|\sigma_{n-1}(a)\right| \cdot\left|\sigma_{n}(a)\right|=\left|\sigma_{n}(a)\right|^{2}>2^{4 n(n-2)}$ and since $n \geq 3,4 n(n-2) \geq 4 n$ and so $\left|\sigma_{n}(a)\right|^{2}>2^{4 n}$, i.e. $\left|\sigma_{n}(a)\right|>2^{2 n}$. 
(2) Since, for $i \leq n-2, \sigma_{i}(a) \in R$ and $\left|\sigma_{i}(a)\right|<1$, we get that $\sigma_{i, j}(\delta) \in i R$. So

$$
\left|\sigma_{i, j}(\varepsilon)\right|^{2}=\left|\sigma_{i}(a)+\sigma_{i, j}(\delta)\right|^{2}=\sigma_{i}(a)^{2}+\left|\sigma_{i, j}(\delta)\right|^{2}=1 .
$$

(3) $\sigma_{n, 1}(\varepsilon)+\sigma_{n, 2}(\varepsilon)=2 \sigma_{n}(a)$, so that we have that

$$
\begin{aligned}
& \left|\sigma_{n, 1}(\varepsilon)\right|+\left|\sigma_{n, 2}(\varepsilon)\right|=\left|\sigma_{n, 1}(\varepsilon)\right|+\left|\sigma_{n, 1}(\varepsilon)\right|^{-1} \\
& \quad \geq\left|\sigma_{n, 1}(\varepsilon)+\sigma_{n, 2}(\varepsilon)\right|=2\left|\sigma_{n}(a)\right|>2^{2 n+1} \quad \text { (by (1)). }
\end{aligned}
$$

So either $\left|\sigma_{n, 1}(\varepsilon)\right|>2^{2 n}$ or $\left|\sigma_{n, 1}(\varepsilon)^{-1}\right|=\left|\sigma_{n, 2}(\varepsilon)\right|>2^{2 n}$. Similarly for $\sigma_{n-1}$.

NOTATIONAL REMARK. From now on we adopt the convention that $\sigma_{n-1,1}$ and $\sigma_{n, 1}$ are such that $\left|\sigma_{n-1,1}(\varepsilon)\right|>1$ and $\left|\sigma_{n, 1}(\varepsilon)\right|>1$.

REMARK. It is well known that if $\varphi(n)$ is the Euler function of $n$ then

$$
\lim _{n \rightarrow \infty} \varphi(n)=\infty
$$

and hence there is only a finite number of roots of unity such that their degrees over $\mathbf{Q}$ is less than or equal to $2 n$. Call $d$ the least common multiple of their orders. It is then obvious that for any root of unity $J \in L, J^{d}=1$.

LEMMA 3. Let $K, a, \delta$ be as above. Let $d$ be as in the last remark. Then all the solutions $(x, y)$ in $O_{K}$ of the equation $x^{2}-\delta^{2} y^{2}=1$, for which there are $x^{*}$ and $y^{*}$ in $O_{K}$ such that $x+\delta y=\left(x^{*}+\delta y^{*}\right)^{6 d}$ and $x^{* 2}-\delta^{2} y^{* 2}=1$, are given by $x= \pm x_{m}$ and $y= \pm y_{m}$ for some $m \in \mathbf{Z}$.

ProOF. By the Dirichlet-Minkowski theorem on units (see [1]), there are $n-2$ fundamental units in $K$. Also $L$ has no real embeddings into $\mathbf{C}$ and so $L$ has $2 n / 2-1=n-1$ fundamental units. Consider the set $S=\left\{x+\delta y \mid x^{2}-\delta^{2} y^{2}=1\right.$, $\left.x, y \in O_{K}\right\} . \quad S$ is clearly in the kernel of the map $N_{L / K}: O_{L} \backslash\{0\} \rightarrow O_{K} \backslash\{0\}$ considered as a multiplicative homomorphism. For any unit $u$ of $O_{K}, N_{L / K}(u)=u^{2}$ and hence the image of $N_{L / K}$ has torsion-free rank at least equal to $n-2$. Therefore, the torsion-free rank of $S$ is at most $(n-1)-(n-2)=1$. Since $\varepsilon$ is in $S$ and $\varepsilon$ is torsion free, rank $S=1$. Hence there is a unit $\varepsilon_{0}=x^{\prime}+\delta y^{\prime} \in S$ such that every $u \in S$ can be written in the form $u=J \varepsilon_{0}^{m}$ where $m \notin \mathbf{Z}$ and $J$ is a root of unity in $L$. In particular $\varepsilon=J_{0} \varepsilon_{0}^{e}$ for some $e \notin \mathbf{Z}, e \neq 0$ and a root of unity $J_{0} \in L$ (so $J_{0}^{d}=1$ ). Clearly we may assume that $e>0$ interchanging $\varepsilon_{0}$ with $\varepsilon_{0}^{-1}$ if necessary. Then $\varepsilon_{0}-\varepsilon_{0}^{-1}=2 \delta y^{\prime}$ and $\varepsilon-\varepsilon^{-1}=2 \delta$, so $\varepsilon-\varepsilon^{-1} \mid \varepsilon_{0}-\varepsilon_{0}^{-1}$. So $|N(2 \delta)| \leq\left|N\left(\varepsilon_{0}-\varepsilon_{0}^{-1}\right)\right|$, where $N=N_{L / Q}$. We have

$$
|N(2 \delta)|=2^{2 n}|N(\delta)|=\left|\prod_{i=1}^{n-2}\left(\sigma_{i}(a)^{2}-1\right)\right| \cdot\left|\sigma_{n}(a)^{2}-1\right|^{2} \cdot 2^{2 n}
$$

since $\sigma_{n}(a)^{2}-1=\overline{\sigma_{n-1}(a)^{2}-1}$. Hence

$$
\begin{aligned}
|N(2 \delta)| & \geq 2^{2 n} \cdot\left(1-1 / 2^{16 n^{2}}\right)^{n-2} \cdot\left|\sigma_{n}(a)^{2}-1\right|^{2}>2^{2 n} \cdot\left(1 / 2^{2}\right)^{n-2} \cdot\left|\sigma_{n}(a)^{2}-1\right|^{2} \\
& =2^{4}\left|\sigma_{n}(a)^{2}-1\right|^{2} \geq\left. 2^{4} \cdot|| \sigma_{n}(a)\right|^{2}-\left.1\left|\geq 2^{3}\right| \sigma_{n}(a)\right|^{2}
\end{aligned}
$$

using $(*)$. Finally

$$
|N(2 \delta)|>2^{2} \cdot\left|\sigma_{n}(a)\right|^{2}(i)
$$


Now observe that $\sigma_{n-1,1}\left(\varepsilon_{0}\right)=\sigma_{n-1}\left(x^{\prime}\right)+\sigma_{n-1,1}(\delta) \sigma_{n-1}\left(y^{\prime}\right)$ and $\sigma_{n-1,2}\left(\varepsilon_{0}\right)=$ $\sigma_{n-1}\left(x^{\prime}\right)+\sigma_{n-1,2}(\delta) \sigma_{n-1}\left(y^{\prime}\right)=\sigma_{n-1}\left(x^{\prime}\right)-\sigma_{n-1,1}(\delta) \sigma_{n-1}\left(y^{\prime}\right)$. So $\sigma_{n-1,2}\left(\varepsilon_{0}\right)=$ $\sigma_{n-1,1}\left(\varepsilon_{0}^{-1}\right)$ and hence

$\left|\sigma_{n-1,1}\left(\varepsilon_{0}\right)-\sigma_{n-1,1}\left(\varepsilon_{0}^{-1}\right)\right| \cdot\left|\sigma_{n-1,2}\left(\varepsilon_{0}\right)-\sigma_{n-1,2}\left(\varepsilon_{0}^{-1}\right)\right|=\left|\sigma_{n-1,1}\left(\varepsilon_{0}\right)-\sigma_{n-1,1}\left(\varepsilon_{0}^{-1}\right)\right|^{2}$.

Similarly for $\sigma_{n, 1}\left(\varepsilon_{0}\right)$ and $\sigma_{n, 2}\left(\varepsilon_{0}\right)$. Moreover,

$$
\left(\sigma_{n, 1}\left(\varepsilon_{0}\right)-\sigma_{n, 1}\left(\varepsilon_{0}^{-1}\right)\right)^{2}=4\left(\sigma_{n}(a)^{2}-1\right) \sigma_{n}\left(y^{\prime}\right)^{2}
$$

and

$$
\left(\sigma_{n-1,1}\left(\varepsilon_{0}\right)-\sigma_{n-1,1}\left(\varepsilon_{0}^{-1}\right)\right)^{2}=4\left(\sigma_{n-1}(a)^{2}-1\right) \sigma_{n-1}\left(y^{\prime}\right)^{2}
$$

and since $\sigma_{n}(a)^{2}=\overline{\sigma_{n-1}(a)^{2}}$ and $\sigma_{n}\left(y^{\prime}\right)^{2}=\overline{\sigma_{n-1}\left(y^{\prime}\right)^{2}}$, we get

$$
\left(\sigma_{n, 1}\left(\varepsilon_{0}\right)-\sigma_{n, 1}\left(\varepsilon_{0}^{-1}\right)\right)^{2}=\overline{\left(\sigma_{n-1,1}\left(\varepsilon_{0}\right)-\sigma_{n-1,1}\left(\varepsilon_{0}^{-1}\right)\right)^{2}} .
$$

Also since $\left|\sigma_{n, 1}\left(\varepsilon_{0}\right)\right|^{e}=\left|\sigma_{n, 1}(\varepsilon)\right|$ and $\left|\sigma_{n, 1}(\varepsilon)\right|>1$, we get $\left|\sigma_{n, 1}\left(\varepsilon_{0}\right)\right|>1$, using the convention $e>0$. Similarly $\left|\sigma_{n-1,1}\left(\varepsilon_{0}\right)\right|>1$. So we get

$$
\begin{aligned}
\left|N\left(\varepsilon_{0}-\varepsilon_{0}^{-1}\right)\right| & =\prod_{\substack{i=1 \\
j=1,2}}^{n}\left|\sigma_{i, j}\left(\varepsilon_{0}\right)-\sigma_{i, j}\left(\varepsilon_{0}\right)^{-1}\right| \leq \\
& \prod_{\substack{i=n-1, n \\
j=1,2}}\left|\sigma_{i, j}\left(\varepsilon_{0}\right)-\sigma_{i, j}\left(\varepsilon_{0}^{-1}\right)\right| \\
& =2^{2 n-4} \cdot\left|\sigma_{n, 1}\left(\varepsilon_{0}\right)-\sigma_{n, 1}\left(\varepsilon_{0}^{-1}\right)\right|^{4}
\end{aligned}
$$

and finally we get

$$
\left|N\left(\varepsilon_{0}-\varepsilon_{0}^{-1}\right)\right| \leq 2^{2 n-4}\left|\sigma_{n, 1}\left(\varepsilon_{0}\right)-\sigma_{n, 1}\left(\varepsilon_{0}\right)^{-1}\right|^{4} .
$$

Now clearly we have

$$
\begin{aligned}
& \left|\sigma_{n, 1}\left(\varepsilon_{0}\right)-\sigma_{n, 1}\left(\varepsilon_{0}\right)^{-1}\right|^{2}=\left|\sigma_{n, 1}\left(\varepsilon_{0}\right)^{2}+\sigma_{n, 1}\left(\varepsilon_{0}\right)^{-2}-2\right| \\
& \quad \leq\left|\sigma_{n, 1}\left(\varepsilon_{0}\right)\right|^{2}+\left|\sigma_{n, 1}\left(\varepsilon_{0}\right)\right|^{-2}+2 \\
& \quad \leq 2\left(\left|\sigma_{n, 1}\left(\varepsilon_{0}\right)\right|^{2}+\left|\sigma_{n, 1}\left(\varepsilon_{0}\right)\right|^{-2}\right)
\end{aligned}
$$

and so

and hence

$$
\left|\sigma_{n, 1}\left(\varepsilon_{0}\right)-\sigma_{n, 1}\left(\varepsilon_{0}\right)^{-1}\right|^{4} \leq 4\left(\left|\sigma_{n, 1}\left(\varepsilon_{0}\right)\right|^{2}+\left|\sigma_{n, 1}\left(\varepsilon_{0}\right)\right|^{-2}\right)^{2}
$$

$$
\left|N\left(\varepsilon_{0}-\varepsilon_{0}^{-1}\right)\right| \leq 2^{2 n-2}\left(\left|\sigma_{n, 1}\left(\varepsilon_{0}\right)^{2}\right|+\left|\sigma_{n, 1}\left(\varepsilon_{0}\right)\right|^{-2}\right)^{2} .
$$

If $|\varepsilon|=\left|\varepsilon_{0}\right|^{e}$ and $e \geq 4$ then $\left|\sigma_{n, 1}(\varepsilon)\right| \geq\left|\sigma_{n, 1}\left(\varepsilon_{0}\right)\right|^{4}>1$ and so

$$
\begin{aligned}
& \left|N\left(\varepsilon_{0}-\varepsilon_{0}^{-1}\right)\right| \leq 2^{2 n-2}\left(\left|\sigma_{n, 1}\left(\varepsilon_{0}\right)\right|^{2}+\left|\sigma_{n, 1}\left(\varepsilon_{0}\right)\right|^{-2}\right)^{2} \\
& \quad=2^{2 n-2}\left(\left|\sigma_{n, 1}\left(\varepsilon_{0}\right)\right|^{4}+\left|\sigma_{n, 1}\left(\varepsilon_{0}\right)\right|^{-4}+2\right) \leq 2^{2 n-1}\left(\left|\sigma_{n, 1}\left(\varepsilon_{0}\right)\right|^{4}+\left|\sigma_{n, 1}\left(\varepsilon_{0}\right)\right|^{-4}\right) \\
& \quad \leq 2^{2 n}\left|\sigma_{n, 1}\left(\varepsilon_{0}\right)\right|^{4} \leq 2^{2 n}\left|\sigma_{n, 1}(\varepsilon)\right|=2^{2 n}\left|\sigma_{n}(a)+\sigma_{n, 1}(\delta)\right| \\
& \quad \leq 2^{2 n}\left(\left|\sigma_{n}(a)\right|+\left|\sigma_{n, 1}(\delta)\right|\right)=2^{2 n}\left(\left|\sigma_{n}(a)\right|+\sqrt{\mid \sigma_{n}(a)-1}\right) \\
& \quad \leq 2^{2 n}\left(\left|\sigma_{n}(a)\right|+2\left|\sigma_{n}(a)\right|\right) \leq 2^{2 n+2}\left|\sigma_{n}(a)\right| .
\end{aligned}
$$

Combining the last inequality with (i) above gives $\left|\sigma_{n}(a)\right|<2^{2 n}$ which contradicts Lemma 2(1). So $e \leq 3$. Therefore, if $x^{* 2}+\delta^{2} y^{* 2}=1$ and $x+\delta y=\left(x^{*}+\delta y^{*}\right)^{6 d}$, since for some $n \in \overline{\mathbf{Z}}, x^{*}+\delta y^{*}=J \varepsilon_{0}^{n}$ and $J^{d}=1$ then $x+\delta y=\varepsilon_{0}^{6 n d}=\varepsilon^{n_{1}}$ and hence $x= \pm x_{n_{1}}$ and $y= \pm y_{n_{1}}$ where $n_{1}=6 n / e$. 
LEMMA 4. Assume that $K, a$ are as above, $h, m \in \mathbf{N}$ and

$$
\left|\sigma_{i}\left(y_{h}\right)\right| \geq \frac{1}{2} \quad \text { for } i=1,2, \ldots, n-2 \quad \text { (condition (1)). }
$$

Then

(1) $\left|\sigma_{n}\left(y_{h}\right)\right|>\left|\sigma_{n, 1}(\varepsilon)\right|^{h} / 4\left|\sigma_{n, 1}(\delta)\right|$ and $\left|\sigma_{n, 1}(\varepsilon)\right|>2^{2 n}$,

(2) $y_{h}\left|y_{m} \Rightarrow h\right| m$ (the first divisibility is meant in $O_{K}$, the second in $Z$ ),

(3) $y_{h}^{2}\left|y_{m} \Rightarrow y_{h}\right| m$ in $O_{K}$.

PROOF. (1) We have proved that $\left|\sigma_{n, 1}(\varepsilon)\right|>2^{2 n}$. It is trivial to see that from this fact the following immediately follows: $\left|\sigma_{n, 1}(\varepsilon)\right|^{h}-\left|\sigma_{n, 1}(\varepsilon)\right|^{-h} \geq\left|\sigma_{n, 1}(\varepsilon)\right|^{h} / \sqrt{2}$ for $h \in N_{0}$. So

$$
\left|\sigma_{n}\left(y_{h}\right)\right|=\frac{\left|\sigma_{n, 1}(\varepsilon)^{h}-\sigma_{n, 1}(\varepsilon)^{-h}\right|}{2\left|\sigma_{n, 1}(\delta)\right|}>\frac{\left|\sigma_{n, 1}(\varepsilon)\right|^{h}}{2 \sqrt{2}\left|\sigma_{n, 1}(\delta)\right|} \geq \frac{\left|\sigma_{n, 1}(\varepsilon)\right|^{h}}{4\left|\sigma_{n, 1}(\delta)\right|} .
$$

(2) Suppose $y_{h} \mid y_{m}$ but $h \nmid m$. Set $m=h q+k$, with $q, k \leq \mathbf{N}$ and $0<k<h$. Lemma 1 yields $y_{m}=x_{k} y_{h q}+x_{h q} y_{k}$. Notice that $y_{h} \mid y_{h q}$, hence $y_{h} \mid x_{h q} y_{k}$. Since $x_{h q}^{2}-\left(a^{2}-1\right) y_{h q}^{2}=1$, the elements $y_{h}$ and $x_{h q}$ are relatively prime. Thus $y_{h} \mid y_{k}$ and $\left|N_{K / \mathbf{Q}}\left(y_{h}\right)\right| \leq\left|N_{K / \mathbf{Q}}\left(y_{k}\right)\right|$. From the Introduction we have that $\sigma_{n-1}\left(y_{h}\right)=\overline{\sigma_{n}\left(y_{h}\right)}$. Also from condition 1 and (1),

$$
\begin{aligned}
\left|N_{K / Q}\left(y_{h}\right)\right| & =\left|\sigma_{n-1}\left(y_{n}\right)\right| \cdot\left|\sigma_{n}\left(y_{h}\right)\right| \cdot \prod_{i \leq n-2}\left|\sigma_{i}\left(y_{h}\right)\right| \geq\left|\sigma_{n}\left(y_{h}\right)\right|^{2} \cdot\left(\frac{1}{2}\right)^{n-2} \\
& >\left(\frac{\left|\sigma_{n, 1}(\varepsilon)\right|^{h}}{4\left|\sigma_{n, 1}(\delta)\right|}\right)^{2} \cdot\left(\frac{1}{2}\right)^{n-2} .
\end{aligned}
$$

Now observe that, for $i \leq n-2, \sigma_{i}\left(x_{k}\right)^{2}-\left(\sigma_{i}(a)^{2}-1\right) \cdot \sigma_{i}\left(y_{k}\right)^{2}=1$ and $\sigma_{i}(a)^{2}<1$. So $\left|\sigma_{i}\left(y_{k}\right)\right|<1$ for $i \leq n-2$. Therefore,

$$
\begin{aligned}
\left|N_{K / \mathbf{Q}}\left(y_{k}\right)\right| & =\left|\sigma_{n-1}\left(y_{k}\right)\right| \cdot\left|\sigma_{n}\left(y_{k}\right)\right| \cdot \prod_{i \leq n-2}\left|\sigma_{i}\left(y_{k}\right)\right|<\left|\sigma_{n}\left(y_{k}\right)\right|^{2} \\
& =\frac{\left|\sigma_{n, 1}\left(\varepsilon^{k}\right)-\sigma_{n, 1}(\varepsilon)^{-k}\right|^{2}}{\left(2\left|\sigma_{n, 1}(\delta)\right|\right)^{2}} \leq\left(\frac{2\left|\sigma_{n, 1}(\varepsilon)\right|^{k}}{2\left|\sigma_{n, 1}(\delta)\right|}\right)^{2} \\
& =\frac{\left|\sigma_{n, 1}(\varepsilon)\right|^{2 k}}{\left|\sigma_{n, 1}(\delta)\right|^{2}}
\end{aligned}
$$

Hence

$$
\left(\frac{1}{2}\right)^{n-2} \frac{\left|\sigma_{n, 1}(\varepsilon)\right|^{2 h}}{4^{2}\left|\sigma_{n, 1}(\delta)\right|^{2}}<\frac{\left|\sigma_{n, 1}(\varepsilon)\right|^{2 k}}{\left|\sigma_{n, 1}(\delta)\right|^{2}}
$$

i.e. $\left|\sigma_{n, 1}(\varepsilon)\right|^{2 h-2 k}<2^{n}$ which contradicts (1) since $h-k \geq 1$. Hence $h \mid m$.

(3) is obvious since $\left(\varepsilon^{l h}-\varepsilon^{-l h}\right) /\left(\varepsilon^{h}-\varepsilon^{-h}\right) \equiv 1 \cdot u \bmod \left(\varepsilon^{h}-\varepsilon^{-h}\right)$ where $u$ is a unit and hence if $y_{h}^{2} \mid y_{m}$, by (2) $h \mid m$, i.e. $m=l h$ for some $l \in \mathbf{N}$, and so $y_{m} / y_{h} \equiv 0 \bmod y_{h}$ which means that $m / h \equiv 0 \bmod y_{h}$, i.e. $m \equiv 0 \bmod y_{h}$.

LEMMA 5. If $K, a$ are as above and $k, j \in \mathbf{N}, m \in \mathbf{N}_{0}$ and $\left|\sigma_{i}\left(x_{m}\right)\right| \geq \frac{1}{2}$ for $i=1, \ldots, n-2$, then if $x_{k} \equiv \pm x_{j} \bmod x_{m}$ we get that $k \equiv \pm j \bmod m$ (the two \pm do not have to correspond).

ProOF. Set $k=2 m q \pm k_{0}, j=2 m h \pm j_{0}$ with $q, h, k_{0}, j_{0} \in \mathbf{N}$ and $k_{0} \leq m$, $j_{0} \leq m$. Lemma 1(9) implies $x_{k} \equiv \pm x_{k_{0}} \bmod x_{m}, x_{j} \equiv \pm x_{j_{0}} \bmod x_{m}$. Hence, it is 
sufficient to prove the lemma for $k \leq m, j \leq m$. Thus suppose $x_{k} \equiv \pm x_{j} \bmod x_{m}$, $k \leq m$ and $j \leq m$. We shall prove that $x_{k}= \pm x_{j}$. Assume $x_{k} \neq \pm x_{j}$; then $\left|N_{K / \mathbf{Q}}\left(x_{m}\right)\right| \leq\left|N_{K / \mathbf{Q}}\left(x_{k} \pm x_{j}\right)\right|$. We may assume without loss of generality that $\left|\sigma_{n}\left(x_{k}\right)\right| \geq\left|\sigma_{n}\left(x_{j}\right)\right|$. Then by the hypothesis of the lemma,

$$
\begin{aligned}
& \left|N_{K / \mathbf{Q}}\left(x_{m}\right)\right|=\left|\sigma_{n}\left(x_{m}\right)\right|^{2} \cdot \prod_{i \leq n-2}\left|\sigma_{i}\left(x_{m}\right)\right| \geq\left|\sigma_{n}\left(x_{m}\right)\right|^{2} \cdot\left(\frac{1}{2}\right)^{n-2} \\
& \quad=\left(\frac{1}{2}\right)^{n-2} \cdot \frac{\left|\sigma_{n, 1}(\varepsilon)^{m}+\sigma_{n, 1}(\varepsilon)^{-m}\right|^{2}}{4} \geq\left(\frac{1}{2}\right)^{n}\left(\left|\sigma_{n, 1}(\varepsilon)\right|^{m}-\left|\sigma_{n, 1}(\varepsilon)\right|^{-m}\right)^{2} \\
& \quad>\left(\frac{1}{2}\right)^{n+1}\left|\sigma_{n, 1}(\varepsilon)\right|^{2 m} .
\end{aligned}
$$

The last inequality holds by Lemma 4(1). Also

$$
\begin{gathered}
\left|N_{K / \mathbf{Q}}\left(x_{k} \pm x_{j}\right)\right| \leq\left(\left|\sigma_{n}\left(x_{k}\right)\right|+\left|\sigma_{n}\left(x_{j}\right)\right|\right)^{2} \cdot \prod_{i \leq n-2}\left(\left|\sigma_{i}\left(x_{k}\right)\right|+\left|\sigma_{i}\left(x_{j}\right)\right|\right) \\
<\left(2\left|\sigma_{n}\left(x_{k}\right)\right|\right)^{2} \cdot 2^{n-2}=\left|\sigma_{n}\left(x_{k}\right)\right|^{2} \cdot 2^{n} \leq\left|\sigma_{n, 1}(\varepsilon)\right|^{2 k} \cdot 2^{n} .
\end{gathered}
$$

So $\left|\sigma_{n, 1}(\varepsilon)\right|^{2 m-2 k}<2^{2 n+1}$, i.e. $\left|\sigma_{n, 1}(\varepsilon)\right|^{m-k}<2^{n+1}<2^{2 n}$ which contradicts Lemma 4(1), if $m \neq k$. So we get $x_{m}=x_{k}$ and hence $x_{m} \mid x_{j}$. So we conclude that $\left|N_{K / \mathbf{Q}}\left(x_{m}\right)\right| \leq\left|N_{K / \mathbf{Q}}\left(x_{j}\right)\right|$. As we proved above,

$$
\left|N_{K / \mathbf{Q}}\left(x_{m}\right)\right| \geq\left(\frac{1}{2}\right)^{n+1}\left|\sigma_{n, 1}(\varepsilon)\right|^{2 m} .
$$

Also

$$
\begin{aligned}
\left|N_{K / \mathbf{Q}}\left(x_{j}\right)\right| & =\prod_{i \leq n-2}\left|\sigma_{i}\left(x_{j}\right)\right| \cdot\left|\sigma_{n}\left(x_{j}\right)\right|^{2} \leq\left|\sigma_{n}\left(x_{j}\right)\right|^{2} \\
& =\frac{\left|\sigma_{n, 1}(\varepsilon)^{j}+\sigma_{n, 1}(\varepsilon)^{-j}\right|^{2}}{4} \leq\left|\sigma_{n, 1}(\varepsilon)\right|^{2 j} .
\end{aligned}
$$

Hence $\left|\sigma_{n, 1}(\varepsilon)\right|^{2 m-2 j} \leq 2^{n+1}$, which by Lemma $4(1)$ can happen only if $2 m-2 j=0$, i.e. $m=j$. So $x_{k}= \pm x_{j}$. If $x_{k}=x_{j}$, then $\varepsilon^{k}+\varepsilon^{-k}=\varepsilon^{j}+\varepsilon^{-j}$, i.e. $\varepsilon^{k}-\varepsilon^{j}=$ $\varepsilon^{-j}-\varepsilon^{-k}$, i.e. $\varepsilon^{-k}\left(1-\varepsilon^{j-k}\right)=\varepsilon^{j}\left(\varepsilon^{k-j}-1\right)$, i.e. $\left(\varepsilon^{k+j}+1\right)\left(\varepsilon^{k-j}-1\right)=0$, i.e. $k= \pm j$. Similarly, if $x_{k}=-x_{j}, \varepsilon^{k}+\varepsilon^{-k}=-\varepsilon^{j}-\varepsilon^{-j}$, i.e. $\left(\varepsilon^{k}+\varepsilon^{j}\right)\left(1+\varepsilon^{-k-j}\right)=0$, i.e. $k= \pm j$.

LEMMA 6. Suppose that $K$ and $a$ are as above with the additional hypothesis that $\sigma_{n, 1}(\varepsilon) / \sigma_{n-1,1}(\varepsilon)$ is not a root of unity. Let $k \in \mathbf{N}_{0}$. Then there exist multiples $m, h$ of $k$ such that $\left|\sigma_{i}\left(x_{m}\right)\right|>\frac{1}{2}$ for $i=1,2, \ldots, n-2$ and $\left|\sigma_{i}\left(y_{h}\right)\right|>\frac{1}{2}$ for $i=1,2, \ldots, n-2$.

PROOF. We shall prove that if

$$
\sigma_{1,1}(\varepsilon)^{k_{1}} \sigma_{2,1}(\varepsilon)^{k_{2}} \cdots \sigma_{n-2,1}(\varepsilon)^{k_{n-2}}=1,
$$

then $k_{1}=k_{2}=\cdots=k_{n-2}=0$. Let $K_{1}$ be the least normal extension of $K$ and $L_{1}$ the least normal extension of $L$, so $K_{1} \subset L_{1}$. It is enough to prove that for each $\sigma_{i}$, $i \leq n-2$, there is an automorphism $\tau$ of $K_{1}$ such that $\tau \sigma_{i}=\sigma_{n-1}$ and $\tau \sigma_{n-1}=\sigma_{i}$ and for all $j \neq i, n-1, \tau \sigma_{j}=\sigma_{j}$, where by $\tau \sigma_{j}$ we mean the restriction of $\tau$ on $\sigma_{j}(K)$ composition $\sigma_{j}$. This is enough because for each $i \leq n-2$, applying the corresponding $\tau$ extended to $L_{1}$ on both sides of (1) and taking absolute values, we get $\left|\sigma_{n, j}(\varepsilon)^{k_{i}}\right|=1$ where $j=1$ or 2 ; hence $k_{i}=0$ and hence the result follows by the theorem of Kronecker (see [5]). 
Notice that every automorphism of $K_{1}$ determines a permutation of the embeddings of $K$ and conversely every permutation of these embeddings determines at most one automorphism of $K_{1}$. So when we write $\tau=\left(\sigma_{i}, \sigma_{j}\right)$ we mean that $\tau$ is the unique automorphism of $K_{1}$ which transposes $\sigma_{i}$ and $\sigma_{j}$. Since $\sigma_{n-1}(K) \neq \sigma_{n}(K)$, the degree of the extension $\sigma_{n-1}(K) \sigma_{n}(K)$ over $\sigma_{n}(K)$ is at least 2, so the identity embedding of $\sigma_{n}(K)$ into $\mathbf{C}$ extends to at least one nonidentity embedding of $\sigma_{n-1}(K) \sigma_{n}(K)$ into $\mathbf{C}$. This embedding extends to an automorphism $\tau_{1}$ of $K_{1}$. Since $\tau_{1}$ is not the identity on $\sigma_{n-1}(K) \sigma_{n}(K)$ and is the identity on $\sigma_{n}(K)$, it can not be the identity on $\sigma_{n-1}(K)$. So, since $\tau_{1} \sigma_{n-1} \neq \sigma_{n-1}$ and $\tau_{1} \sigma_{n-1} \neq \sigma_{n}$, $\tau_{1} \sigma_{n-1}$ is a real embedding of $K$, say $\tau_{1} \sigma_{n-1}=\sigma_{i_{0}}$. Let $\tau_{0}$ be the automorphism of $K_{1}$ such that $\tau_{0}(x)=\bar{x}$. Then $\tau_{1} \tau_{0} \tau_{1}^{-1}=\left(\sigma_{i_{0}}, \sigma_{n}\right)$, since $\tau_{0}$ is a transposition $\left(\tau_{0}=\left(\sigma_{n}, \sigma_{n-1}\right)\right)$.

Now assume that $\sigma_{n-1}(K) \subset \sigma_{1}(K) \cdots \sigma_{n-2}(K) \sigma_{n}(K)$. Applying $\tau_{1} \tau_{0} \tau_{1}^{-1}$ to both sides we find $\sigma_{n-1}(K) \subset \sigma_{1}(K) \cdots \sigma_{n-2}(K)$ which is impossible since $\sigma_{n-1}(K)$ is nonreal and the right-hand side of the relation is real. So

$$
\sigma_{n-1}(K) \not \subset \sigma_{1}(K) \cdots \sigma_{n-2}(K) \sigma_{n}(K) .
$$

Let $i \leq n-2$. Consider the extension

$$
\sigma_{n-1}(K) \sigma_{1}(K) \cdots \sigma_{i-1}(K) \sigma_{i+1}(K) \cdots \sigma_{n-2}(K) \sigma_{n}(K)
$$

over $\sigma_{1}(K) \cdots \sigma_{i-1}(K) \sigma_{i+1}(K) \cdots \sigma_{n-2}(K) \sigma_{n}(K)$. This extension may not be of degree 1 , otherwise $\sigma_{n-1}(K) \subset \sigma_{1}(K) \cdots \sigma_{i-1}(K) \sigma_{i+1}(K) \cdots \sigma_{n-2}(K) \sigma_{n}(K)$, contrary to what we proved. So the identity embedding in $\mathbf{C}$ of the ground field extends to at least one nonidentity embedding of the extension field in $\mathbf{C}$. Let $\tau$ be an extension of this embedding to an automorphism of $K_{1}$. Clearly, since $\tau \sigma_{n-1} \neq \sigma_{n-1}$ and $\tau \sigma_{j}=\sigma_{j}$ for $j \neq i, n-1$, we must have $\tau \sigma_{n-1}=\sigma_{i}$ and hence $\tau=\left(\sigma_{i}, \sigma_{n-1}\right)$ and this is what we should prove in order to conclude the lemma.

LEMMA 7. Suppose that $K$ and $a$ are as above and that $\left|\sigma_{i}(a)\right|<1 / 2^{8 n}$ for $i=1,2, \ldots, n-2$. Let $m \in \mathbf{N}_{0}$. Then there exists an element $b$ in $O_{K}$ such that:

(1) $b \equiv 1 \bmod y_{m}(a)$

(2) $b \equiv a \bmod x_{m}(a)$;

(3) b satisfies $(*)$.

PROOF. Set $b=x_{m}^{2 s}+a\left(1-x_{m}^{2}\right)$ with $s \in \mathbf{N}_{0}$ to be determined. Since $x_{m}^{2}-$ $\left(a^{2}-1\right) y_{m}^{2}=1$, we have $x_{m}^{2} \equiv 1 \bmod y_{m}$; hence (1) holds. Also (2) holds obviously. Since $\left|\sigma_{i}\left(x_{m}\right)\right|<1$ for $i=1,2, \ldots, n-2$ and $\left|\sigma_{n}\left(x_{m}\right)\right| \cdot\left|\sigma_{n-1}\left(x_{m}\right)\right|=\left|\sigma_{n}\left(x_{m}\right)\right|^{2}>1$, we can choose $s$ large enough so that $\left|\sigma_{i}\left(x_{m}\right)^{2 s}\right|<1 / 2^{8 n}$ for $i=1,2, \ldots, n-2$. Then for $i=1,2, \ldots, n-2$ the following holds:

$$
\left|\sigma_{i}(b)\right| \leq\left|\sigma_{i}\left(x_{m}\right)^{2 s}\right|+\left|\sigma_{i}(a)\right| \cdot\left|1-\sigma_{i}\left(x_{m}\right)^{2}\right|<\left|\sigma_{i}\left(x_{m}\right)\right|^{2 s}+\frac{1}{2^{8 n}}<\frac{2}{2^{8 n}}<\frac{1}{2^{4 n}} .
$$

LEMMA 8. Let $K$ be any number field of degree $n$ over $\mathbf{Q}$, and let $\sigma_{1}, \sigma_{2}, \ldots, \sigma_{n}$ be the embeddings of $K$ into C. Let $\xi, z \in O_{K}$ and $z \neq 0$. If $2^{n+1} \xi^{n}(\xi+1)^{n} \ldots$ $(\xi+n-1)^{n} \mid z$, then $\left|\sigma_{i}(\xi)\right|<\frac{1}{2}|N(z)|^{1 / n}$ for all $i=1,2, \ldots, n$.

ProOF. See [3]. 
MAIN LEMMA. Let $K$ be as above and $a \in O_{K}$ satisfying $\left|\sigma_{i}(a)\right|<1 / 2^{8 n}$ for $i=1,2, \ldots, n-2$. (**)

and let $d$ be defined as in the Remark before Lemma 3. Define the subset $S$ of $O_{K}$ by $\xi \in S \Leftrightarrow \xi \in O_{K} \wedge \exists x, y, w, z, u, v, s, t, x^{\prime}, y^{\prime}, w^{\prime}, z^{\prime}, u^{\prime}, v^{\prime}, s^{\prime}, t^{\prime}, b$ in $O_{K}$ :

$$
\begin{aligned}
& x^{\prime 2}-\left(a^{2}-1\right) y^{2}=1, \\
& w^{\prime 2}-\left(a^{2}-1\right) z^{2}=1 \text {, } \\
& u^{\prime 2}-\left(a^{2}-1\right) v^{2}=1 \text {, } \\
& s^{2}-\left(b^{2}-1\right) t^{\prime 2}=1, \\
& x+\delta(a) y=\left(x^{\prime}+\delta(a) y^{\prime}\right)^{6 d}, \\
& w+\delta(a) z=\left(w^{\prime}+\delta(a) z^{\prime}\right)^{6 d}, \\
& u+\delta(a) v=\left(u^{\prime}+\delta(a) v^{\prime}\right)^{6 d} \text {, } \\
& s+\delta(b) t=\left(s^{\prime}+\delta(b) t^{\prime}\right)^{6 d}, \\
& \left|\sigma_{i}(b)\right|<1 / 2^{4 n}, \quad i=1,2, \ldots, n-2, \\
& \left|\sigma_{i}(z)\right| \geq \frac{1}{2}, \quad i=1,2, \ldots, n-2, \\
& \left|\sigma_{i}(u)\right| \geq \frac{1}{2}, \quad i=1,2, \ldots, n-2, \\
& v \neq 0 \text {, } \\
& z^{2} \mid v \\
& b \equiv 1 \bmod z \text {, } \\
& b \equiv a \bmod u \text {, } \\
& s \equiv x \bmod u \text {, } \\
& t \equiv \xi \bmod z, \\
& 2^{n+1} \xi^{n}(\xi+1)^{n} \cdots(\xi+n-1)^{n} x^{n}(x+1)^{n} \cdots(x+n-1)^{n} \mid z .
\end{aligned}
$$

Then $N_{0} \subset S \subset \mathbf{Z}$.

ProOF. (i) Suppose there are $x, y, \ldots, b \in O_{K}$ satisfying (1)-(14). We shall prove that $\xi \in \mathbf{Z}$. From $(* *)$ and (5) it follows that $a$ and $b$ satisfy $(*)$. Hence from (1)-(4), $\left(1^{*}\right)-\left(4^{*}\right)$ and Lemma 3 it follows that there are $k, h, m, j \in \mathrm{N}$ such that:

$$
\begin{aligned}
x & = \pm x_{k}(a), & & y= \pm y_{k}(a), \\
w & = \pm x_{h}(a), & & z= \pm y_{h}(a), \\
u & = \pm x_{m}(a), & & v= \pm y_{m}(a), \\
s & = \pm x_{j}(b), & & t= \pm y_{j}(b) .
\end{aligned}
$$

So (6)-(13) become

$$
\begin{aligned}
\left|\sigma_{i}\left(y_{h}(a)\right)\right| \geq & \frac{1}{2} \quad \text { for } i=1,2, \ldots, n-2, \\
\left|\sigma_{i}\left(x_{m}(a)\right)\right| \geq & \frac{1}{2} \quad \text { for } i=1,2, \ldots, n-2, \\
& y_{m}(a) \neq 0 \\
& y_{h}^{2}(a) \mid y_{m}(a) \\
& b \equiv 1 \bmod y_{h}(a) \\
& b \equiv a \bmod x_{m}(a)
\end{aligned}
$$


We have

$$
\begin{gathered}
y_{j}(b) \equiv j \bmod (b-1) \quad(\text { Lemma } 1(7)) \\
y_{j}(b) \equiv j \bmod y_{h}(a) \quad\left(\text { by }\left(10^{\prime}\right)\right), \\
j \equiv \pm \xi \bmod y_{h}(a) \quad\left(\text { by }\left(13^{\prime}\right)\right) \\
x_{j}(b) \equiv x_{j}(a) \bmod x_{m}(a) \quad\left(\text { by }\left(11^{\prime}\right) \text { and Lemma } 1(8)\right) \\
x_{j}(a) \equiv \pm x_{k}(a) \bmod x_{m}(a) \quad\left(\text { by }\left(12^{\prime}\right)\right) \\
k \equiv \pm j \bmod m \quad\left(\text { by }\left(7^{\prime}\right),\left(8^{\prime}\right) \text { and Lemma } 5\right), \\
y_{h}(a) \mid m \quad\left(\text { by }\left(6^{\prime}\right),\left(9^{\prime}\right) \text { and Lemma } 4\right), \\
k \equiv \pm j \bmod y_{h}(a) \quad(\text { by }(16)), \\
k \equiv \pm \xi \bmod z \quad(\text { by }(15)), \\
\left|\sigma_{i}(\xi)\right|<\frac{1}{2}|N(z)|^{1 / n} \quad \text { for } i=1,2, \ldots, n \quad(\text { by }(14) \text { and Lemma } 8), \\
k<\left|\sigma_{n}\left(x_{k}(a)\right)\right|<\frac{1}{2}|N(z)|^{1 / n} \quad(\text { by }(14) \text { and Lemma } 8), \\
\left|\sigma_{i}(k \pm \xi)\right|<|N(z)|^{1 / n} \quad \text { for } i=1,2, \ldots, n .
\end{gathered}
$$

So $|N(k+\xi)|<|N(z)|$ and so $k= \pm \xi$ (by (17)).

(ii) Conversely, suppose $\xi \in \mathbf{N}_{0}$. We shall prove that there are $x, y, \ldots, b \in O_{K}$ satisfying (1)-(14). Set $k=\xi \in \mathbf{N}_{0}, x^{\prime}=x_{k}(a)$ and $y^{\prime}=y_{k}(a)$; then (1) and $\left(1^{*}\right)$ are satisfied. By Lemmas $1(10),(4)$ and 6 there exists an $h \in \mathbf{N}_{0}$ such that the left-hand side of $(14)$ divides $y_{h}(a)$ and $\left|\sigma_{i}\left(y_{h}(a)\right)\right| \geq \frac{1}{2}$ for $i=1,2, \ldots, n-2$. Set $w^{\prime}=x_{h}(a)$ and $z=y_{h}(a)$, then (2), (6) and (14) are satisfied. Again by Lemmas 1(10), (4) and 6, there exists an $m \in \mathbf{N}_{0}$ such that $y_{h}^{2}(a) \mid y_{m}(a)$ and $\left|\sigma_{i}\left(x_{m}(a)\right)\right| \geq \frac{1}{2}$ for $i=1,2, \ldots, n-2$. Set $u^{\prime}=x_{m}(a)$ and $v^{\prime}=y_{m}(a)$; then (3), $\left(3^{*}\right)$ and $(7)-(9)$ are satisfied. From Lemma 7 it follows that there exists $b \in O_{K}$ satisfying (10), (11) and (5). Set $s^{\prime}=x_{k}(b)$ and $t^{\prime}=y_{k}(b)$; then (4) is satisfied. Lemma 1(8) and (11) imply (12) and Lemma 1(7) and (10) imply (13). Thus all conditions are satisfied and $\xi \in S$.

LEMMA 9. Let $K$ be any number field.

(i) If $R_{1}$ and $R_{2}$ are diophantine relations over $O_{K}$, then $R_{1} \vee R_{2}$ and $R_{1} \wedge R_{2}$ are also diophantine over $O_{K}$.

(ii) The relation $x \neq 0$ is diophantine over $O_{K}$.

ProOF. See [3].

LEMMA 10. Let $K$ be any number field, and $\sigma$ an embedding of $K$ into $\mathbf{R}$. Then the relation $\sigma(x) \geq 0$ is diophantine over $O_{K}$.

PROOF. See [3].

THEOREM. Let $K$ be a number field with exactly two nonreal embeddings into $\mathbf{C}$, of degree $n \geq 3$ over $\mathbf{Q}$. Then $\mathbf{Z}$ is diophantine over $O_{K}$.

PROOF. By Minkowski's lemma on convex bodies it follows that there is an $a$ satisfying $(* *)$ of the Main Lemma. By Lemma 10 the relations (5)-(7) are 
diophantine over $O_{K}$ and clearly the relations $\left(1^{*}\right)-\left(4^{*}\right)$ can be written so that $\delta(a)$ and $\delta(b)$ do not occur, i.e. $\left(1^{*}\right)-\left(4^{*}\right)$ are diophantine over $O_{K}$. So the set $S$ of the Main Lemma is diophantine over $L_{K}$ and hence $\mathbf{Z}$ is also diophantine over $O_{K}$.

\section{REFERENCES}

1. Z. I. Borevich and I. R. Shafarevich, Number theory, "Nauka", Moskow, 1964; English transl., Pure Appl. Math. 20, Academic Press, New York 1966.

2. J. Denef, Hilbert's Tenth Problem for quadratic rings, Proc. Amer. Math. Soc. 48 (1975), 214220.

3. J. Denef, Diophantine sets over algebraic integer rings. II, Trans. Amer. Math. Soc. 257 (1980).

4. J. Denef and L. Lipshitz, Diophantine sets over some rings of algebraic integers, J. London Math. Soc. (2) 18 (1978), 385-391.

5. G. Hardy and E. Wright, An introduction to the theory of numbers, Oxford Univ. Press, Oxford, 1960.

6. Yu. Matijasevic, Enumerable sets are diophantine, Dokl. Akad. Nauk SSSR 191 (1970), 272282; English transl., Soviet Math. Dokl. 11 (1970), 354-357.

Computer Technology institute, Patras 26110, Greece

Current address: Department of Mathematics, Florida International University, University Park, Miami, Florida 33199 